\title{
Hopfian and strongly hopfian manifolds
}

\author{
by \\ Young Ho Im (Pusan) and \\ Yongkuk Kim (Pusan and Knoxville, Tenn.)
}

\begin{abstract}
Let $p: M \rightarrow B$ be a proper surjective map defined on an $(n+2)$-manifold such that each point-preimage is a copy of a hopfian $n$-manifold. Then we show that $p$ is an approximate fibration over some dense open subset $O$ of the $\bmod 2$ continuity set $C^{\prime}$ and $C^{\prime} \backslash O$ is locally finite. As an application, we show that a hopfian $n$-manifold $N$ is a codimension-2 fibrator if $\chi(N) \neq 0$ or $H_{1}(N) \cong \mathbb{Z}_{2}$.
\end{abstract}

1. Introduction. Call a closed manifold $N$ hopfian if it is orientable and every degree one map $N \rightarrow N$ which induces a $\pi_{1}$-isomorphism is a homotopy equivalence. A closed $n$-manifold $N$ is called a strongly hopfian manifold if $N_{H}$ is hopfian, where $H$ is the intersection of all subgroups with index 2 in $\pi_{1}(N)$ and $N_{H}$ is the covering space of $N$ corresponding to $H$.

In [16] and [17], using the concept of strong hopfianness, Y. Kim showed that all closed strongly hopfian manifolds $N$ with residually finite $\pi_{1}(N)$ and $\chi(N) \neq 0$ as well as all closed strongly hopfian manifolds with hyperhopfian fundamental group are codimension-2 fibrators. It is well known that every finitely generated residually finite group is hopfian. While every subgroup of a residually finite group is residually finite, a hopfian group can contain non-hopfian subgroups of finite index. For example, consider the group $G=\left\langle a, b: a^{-1} b^{12} a=b^{18}\right\rangle$ and a homomorphism $f: G \rightarrow \mathbb{Z}_{2}$ given by $f(a)=0, f(b)=1$. By the Reidemeister-Schreier method, one can see that the kernel $K$ of $f$ is isomorphic to $\left\langle c_{1}, c_{2}, d: c_{i}^{-1} d^{6} c_{i}=d^{9}, i=1,2\right\rangle$, and $[G: K]=2$. Baumslag-Solitar [1] tells us that $G$ is hopfian but $K$

1991 Mathematics Subject Classification: Primary 57N15, 55R65; Secondary 57N25, $54 \mathrm{~B} 15$.

Key words and phrases: approximate fibration; codimension-2 fibrator; hopfian manifold; strongly hopfian manifold; hyperhopfian group; degree one mod 2 map; continuity set.

The first author was supported by the Basic Science Research Institute Program, Ministry of Education, 1997. 
is non-hopfian. Put $H=\bigcap\left\{H_{i} \leq G:\left[G: H_{i}\right]=2\right\}$. Since $[K: H]$ is finite and $K$ is non-hopfian, $H$ is non-hopfian (see [14]). Of course, this example does not give us the existence of a non-strongly hopfian manifold (see [12]). Consequently, the hopfianness of neither $N$ nor $\pi_{1}(N)$ guarantees the hopfianness of $N_{H}$ and $\pi_{1}\left(N_{H}\right)$.

In this paper, we show that if $p: M \rightarrow B$ is a proper surjective map defined on an $(n+2)$-manifold such that each point-preimage is a copy of a hopfian $n$-manifold, then $p$ is an approximate fibration over some dense open subset $O$ of the mod 2 continuity set $C^{\prime}$ and $C^{\prime} \backslash O$ is locally finite. This property enables us to show that, whether or not $N_{H}$ and $\pi_{1}\left(N_{H}\right)$ are hopfian, all hopfian manifolds $N$ with hopfian $\pi_{1}(N)$ and $\chi(N) \neq 0$ are codimension-2 fibrators. Moreover, we can give an affirmative answer to the following question of Chinen [3]: Is every hopfian manifold $N$ with hopfian fundamental group and $H_{1}(N) \cong \mathbb{Z}_{2}$ a codimension-2 fibrator?

2. Preliminaries. Throughout this paper, the symbols $\sim, \approx$, and $\cong$ denote homotopy equivalence, homeomorphism, and isomorphism, respectively. The symbol $\chi$ is used to denote Euler characteristic. All manifolds are understood to be finite-dimensional, connected, metric, and boundaryless. Whenever the presence of boundary is tolerated, the object will be called a manifold with boundary.

A proper map $p: M \rightarrow B$ between locally compact ANRs is called an approximate fibration if it has the approximate homotopy lifting property (see [4]).

A proper map $p: M \rightarrow B$ is $N^{n}$-like if each fiber $p^{-1}(b)$ is shape equivalent to $N$. For simplicity, we shall assume that each fiber $p^{-1}(b)$ in an $N^{n}$-like map is an ANR having the homotopy type of $N^{n}$.

Let $N$ and $N^{\prime}$ be closed $n$-manifolds and $f: N \rightarrow N^{\prime}$ be a map. If both $N$ and $N^{\prime}$ are orientable, then the degree of $f$ is the nonnegative integer $d$ such that the induced endomorphism $f_{*}: H_{n}(N ; \mathbb{Z}) \cong \mathbb{Z} \rightarrow H_{n}\left(N^{\prime} ; \mathbb{Z}\right) \cong \mathbb{Z}$ amounts to multiplication by $d$, up to sign. In general, the degree mod 2 of $f$ is the nonnegative integer $d$ such that the induced endomorphism $f_{*}: H_{n}\left(N ; \mathbb{Z}_{2}\right) \cong \mathbb{Z}_{2} \rightarrow H_{n}\left(N^{\prime} ; \mathbb{Z}_{2}\right) \cong \mathbb{Z}_{2}$ amounts to multiplication by $d$.

Suppose that $N$ is a closed $n$-manifold and a proper map $p: M \rightarrow B$ is $N$-like. Let $G$ be the set of all fibers, i.e., $G=\left\{p^{-1}(b): b \in B\right\}$. Put $C=\left\{p(g) \in B: g \in G\right.$ and there exist a neighborhood $U_{g}$ of $g$ in $M$ and a retraction $R_{g}: U_{g} \rightarrow g$ such that $R_{g} \mid g^{\prime}: g^{\prime} \rightarrow g$ is a degree one map for all $g^{\prime} \in G$ in $\left.U_{g}\right\}$, and $C^{\prime}=\{p(g) \in B: g \in G$ and there exist a neighborhood $U_{g}$ of $g$ in $M$ and a retraction $R_{g}: U_{g} \rightarrow g$ such that $R_{g} \mid g^{\prime}: g^{\prime} \rightarrow g$ is a degree one mod 2 map for all $g^{\prime} \in G$ in $U_{g}$. Call $C$ the continuity set of $p$ 
and $C^{\prime}$ the mod 2 continuity set of $p$. D. Coram and P. Duvall [6] showed that $C$ and $C^{\prime}$ are dense, open subsets of $B$.

A group $\Gamma$ is said to be hopfian if every epimorphism $f: \Gamma \rightarrow \Gamma$ is necessarily an isomorphism. A finitely presented group $\Gamma$ is said to be $h y$ perhopfian if every homomorphism $f: \Gamma \rightarrow \Gamma$ with $f(\Gamma)$ normal and $\Gamma / f(\Gamma)$ cyclic is an isomorphism (onto). A group $\Gamma$ is said to be residually finite if for any non-trivial element $x$ of $\Gamma$ there is a homomorphism $f$ from $\Gamma$ onto a finite group $K$ such that $f(x) \neq 1_{K}$.

A closed $n$-manifold $N^{n}$ is a codimension-2 fibrator (respectively, a codimension-2 orientable fibrator) if, whenever $p: M \rightarrow B$ is a proper map from an arbitrary (respectively, orientable) $(n+2)$-manifold $M$ to a 2-manifold $B$ such that each $p^{-1}(b)$ is shape equivalent to $N$, then $p$ : $M \rightarrow B$ is an approximate fibration.

All simply connected manifolds, closed surfaces with non-zero Euler characteristic, and closed manifolds $N$ with $\pi_{1}(N) \cong \mathbb{Z}_{2}$ (for example, real projective $n$-spaces, $n>1$ ), are known to be codimension-2 fibrators (see [7]).

The following is basic for investigating codimension-2 fibrators.

Proposition 2.1 [7, Proposition 2.8]. If $p: M \rightarrow B$ is a proper surjective map defined on an orientable $(n+2)$-manifold $M$ with closed orientable $n$-manifolds as point inverses, then $B$ is a 2-manifold and $D=B \backslash C$ is locally finite in $B$, where $C$ represents the continuity set of $p$. Moreover, if either $M$ or some point inverses are non-orientable, then $B$ is a 2-manifold with boundary (possibly empty) and $D^{\prime}=($ int $B) \backslash C^{\prime}$ is locally finite in $B$, where $C^{\prime}$ represents the mod 2 continuity set of $p$.

The next result summarizes useful information connecting hopfian manifolds and hopfian fundamental groups.

Proposition 2.2 ([8, Theorem 2.2] or [11]). A closed orientable $n$-manifold $N$ is a hopfian manifold if any one of the following conditions holds:

(1) $n \leq 4$

(2) $\pi_{1}(N)$ is virtually nilpotent;

(3) $\pi_{i}(N)$ is trivial for $1<i<n-1$.

The following two recent facts play important roles in this paper.

Lemma 2.3 [17, Lemma 3.2]. Let $N$ be a closed manifold. Suppose that $f: \pi_{1}(N) \rightarrow \pi_{1}(N)$ is a homomorphism whose induced action on $H_{1}\left(N ; \mathbb{Z}_{2}\right)$ is an automorphism (i.e., $f(H) \subset H$ and the natural map $f^{\prime}: \pi_{1}(N) / H \rightarrow$ $\pi_{1}(N) / H$ is an isomorphism). Then

(1) $f$ is an epimorphism if and only if $f \mid H: H \rightarrow H$ is an epimorphism.

(2) $f$ is an isomorphism if and only if $f \mid H: H \rightarrow H$ is an isomorphism. 
Proposition 2.4 [2, Corollary 3.3]. Let $N$ be a codimension-2 orientable fibrator. If $N$ has no 2-to-1 covering, then $N$ is a codimension-2 fibrator.

\section{Hopfian manifolds as codimension-2 fibrators}

THEOREM 3.1. Let $N$ be a hopfian $n$-manifold with hopfian fundamental group. Let a proper map $p: M \rightarrow B$ defined on an $(n+2)$-manifold $M$ be $N$-like. Then $p$ is an approximate fibration over some dense open subset $O$ of the mod 2 continuity set $C^{\prime}$ of $p$ and $C^{\prime} \backslash O$ is locally finite.

Proof. Let $G=\left\{p^{-1}(b) \equiv g_{b}: b \in B\right\}$.

Claim. Any $x \in C^{\prime}$ has a neighborhood $V_{x}$ and a dense open subset $O_{x}$ of $V_{x}$ such that $p$ is an approximate fibration over $O_{x}$ and $V_{x} \backslash O_{x}$ is locally finite.

Fix $g_{0} \in G$ with $p\left(g_{0}\right) \in C^{\prime}$. Take a neighborhood $U\left(\subset C^{\prime}\right)$ of $p\left(g_{0}\right)$ such that $p^{-1}(U)$ retracts to $g_{0}$, and take a smaller connected neighborhood $V$ of $p\left(g_{0}\right)$ such that $p^{-1}(V)$ deformation retracts to $g_{0}$ in $p^{-1}(U)$. Call this retraction $R: p^{-1}(V) \rightarrow g_{0}$. If $N$ has no 2-to-1 covering, the claim follows from [2, Proposition 3.2] and [8, Theorem 2.1]. Now we assume that $N$ has a 2-to-1 covering. Take the covering map $q: M^{*} \rightarrow p^{-1}(V)$ corresponding to $R_{\#}^{-1}(H)$, where $H=\bigcap_{i \in I} H_{i}$ with $I=\left\{i:\left[\pi_{1}(N): H_{i}\right]=2\right\}$. Since $\left[\pi_{1}\left(p^{-1}(V)\right): R_{\#}^{-1}(H)\right]=\left[\pi_{1}\left(g_{0}\right): H\right]<\infty, q$ is finite. We see that for all $g \in G$ with $p(g) \in C^{\prime}, q^{-1}(g) \equiv g^{*}$ is connected and has homotopy type of $N_{H}$ (see [16, Lemma 3.1] for a detailed proof), where $N_{H}$ is the covering space of $N$ corresponding to $H$. Set $G^{*}=\left\{g^{*}: g \in G\right.$ with $\left.p(g) \in V\right\}$. Let $p^{*}=p \circ q: M^{*} \rightarrow B^{*}=M^{*} / G^{*}=V$ be the composition map. By Proposition 2.1, we see that the continuity set $C\left(p^{*}\right)$ of $p^{*}$ is dense open in $V$, and $V \backslash C\left(p^{*}\right)$ is locally finite. So it is enough to show that $p^{*}$ is an approximate fibration over the continuity set $C\left(p^{*}\right)$ of $p^{*}$.

Fix $g_{b}^{*} \in G^{*}$ with $p^{*}\left(g_{b}^{*}\right)=p\left(g_{b}\right)=b \in C\left(p^{*}\right)$. Carefully take a small neighborhood $W\left(\subset C\left(p^{*}\right)\right)$ of $b$ and a retraction $R_{b}: p^{-1}(W) \rightarrow g_{b}$. Let $R_{b}^{*}: W^{*} \equiv q^{-1}\left(p^{-1}(W)\right) \rightarrow g_{b}^{*}$ be the lifting of $R_{b}$.

For any $a \in W$, consider the diagram

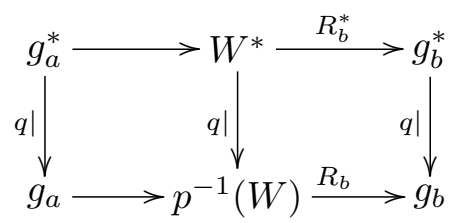

Since $\left(R_{b}^{*} \mid\right): g_{a}^{*} \rightarrow g_{b}^{*}$ is a map of degree one, $\left(R_{b} \mid\right): g_{a} \rightarrow g_{b}$ has degree one. The hopfian hypotheses of $N$ and $\pi_{1}(N)$ yield that $\left(R_{b} \mid\right)$ is a homotopy equivalence. In particular, $\left(R_{b} \mid\right)_{\#}: \pi_{1}\left(g_{a}\right) \rightarrow \pi_{1}\left(g_{b}\right)$ is an isomorphism. 
By Lemma 2.3, we see that $\left(R_{b}^{*} \mid\right)_{\#}: \pi_{1}\left(g_{a}^{*}\right) \rightarrow \pi_{1}\left(g_{b}^{*}\right)$ is an isomorphism. Moreover, since for $i \geq 2$, the homomorphism

$$
\pi_{i}\left(g_{a}^{*}\right) \cong \pi_{i}\left(g_{a}\right) \underset{\left(R_{b} \mid\right)_{\#}}{\cong} \pi_{i}\left(g_{b}\right) \cong \pi_{i}\left(g_{b}^{*}\right)
$$

is an isomorphism, by the Whitehead Theorem $\left(R_{b}^{*} \mid\right)$ is a homotopy equivalence. It follows from [8, Theorem 2.1] and [4] that $p^{*}=p \circ q$ is an approximate fibration over the continuity set $C\left(p^{*}\right)$ of $p^{*}$.

Now let $O=\bigcup_{x \in C^{\prime}} O_{x}$ and $C^{\prime}=\bigcup_{x \in C^{\prime}} V_{x}$. Then we are done.

Remark 1. The conclusion of Theorem 3.1 is best possible, in the following sense: there are proper maps from $S^{1} \times \mathbb{R}^{2} \rightarrow \mathbb{R}^{2}$ with fiber $S^{1}$ which are not approximate fibrations over $C^{\prime}=\mathbb{R}^{2}$ (see [6] or [7, Example 3.6]).

REMARK 2 . Let $N$ be a hopfian $n$-manifold with some properties. The most common procedure of showing that $N$ is a codimension- 2 fibrator can be described as follows: Take any $N$-like proper map $p: M \rightarrow B$ from an $(n+2)$-manifold onto a 2-manifold. First show that $p$ is an approximate fibration over the $\bmod 2$ continuity set $C^{\prime}$ of $p$, and then show that $p$ is an approximate fibration over int $B$ and $\partial B=\emptyset$. The usefulness of Theorem 3.1 is that, showing that $p$ is an approximate fibration over the mod 2 continuity set $C^{\prime}$ of $p$, we can localize the situation so that $C^{\prime}$ is an open disk containing $b_{0}=p\left(g_{0}\right)$ and $p$ is an approximate fibration over $C^{\prime} \backslash b$. Also, we may assume that $R: p^{-1}\left(C^{\prime}\right) \rightarrow g_{0}$ is a strong deformation retraction.

Corollary 3.2. Let $N$ be a hopfian $n$-manifold with hopfian $\pi_{1}(N)$. Then $N$ is a codimension-2 fibrator if

(1) $\chi(N) \neq 0$, or

(2) $H_{1}(N) \cong \mathbb{Z}_{2}$.

Proof. Let a proper map $p: M \rightarrow B$ from an $(n+2)$-manifold onto a 2-manifold with boundary be $N$-like. Set $G=\left\{p^{-1}(b): b \in B\right\}$.

Proof of (1). Applying the method of the proof of [16, Theorem 3.3] to $p \mid C^{\prime}$, we see that $p$ is an approximate fibration over the mod 2 continuity set $C^{\prime}$ of $p$. Then copy the proofs of [16, Lemma 3.2] and [16, Theorem 3.3].

Proof of (2)

Claim (i). $p$ is an approximate fibration over the mod 2 continuity set $C^{\prime}$ of $p$.

Localize the situation so that $C^{\prime}$ is an open disk containing $b_{0}=p\left(g_{0}\right)$ and $p$ is an approximate fibration over $C^{\prime} \backslash b_{0}$. Also, we may assume that $R: p^{-1}\left(C^{\prime}\right) \rightarrow g_{0}$ is a strong deformation retraction. If for any $g \in G$ with $p(g) \in C^{\prime},(R \mid)_{\#}: \pi_{1}(g) \rightarrow \pi_{1}\left(g_{0}\right)$ is an epimorphism, we are done (see [5]). So now assume that there is a $g\left(\neq g_{0}\right) \in G$ with $p(g) \in C^{\prime}$ such 
that $(R \mid)_{\#}: \pi_{1}(g) \rightarrow \pi_{1}\left(g_{0}\right)$ is not an epimorphism. Take the covering $q: M^{*} \rightarrow p^{-1}\left(C^{\prime}\right)$ corresponding to $R_{\#}^{-1}(H)$, where $H=\bigcap_{i \in I} H_{i}$ with $I=\left\{i:\left[\pi_{1}(N): H_{i}\right]=2\right\}$. Here note that $H$ is the commutator subgroup of $\pi_{1}(N)$, for $H_{1}(N)=\mathbb{Z}_{2}$. From the fact that $\pi_{1}\left(g_{0}\right) /(R \mid)_{\#}\left(\pi_{1}(g)\right)$ is cyclic, we see that $(R \mid)_{\#}\left(\pi_{1}(g)\right)=H$, which contradicts the fact that $(R \mid)_{\#}^{-1}(H)=H$ (see [17, Lemma 3.1]).

Claim (ii). $p$ is an approximate fibration over int $B$.

In light of Proposition 2.1, we localize the situation so that int $B$ is an open disk containing $b_{0}=p\left(g_{0}\right)$ and $p$ is an approximate fibration over int $B \backslash b_{0}$. Also, we may assume that $R: p^{-1}$ (int $\left.B\right) \rightarrow g_{0}$ is a strong deformation retraction. It suffices to show that for any $g \in G,(R \mid)_{*}: H_{1}(g) \rightarrow$ $H_{1}\left(g_{0}\right)$ is an isomorphism (see [8, Lemma 5.2] or [15]). So now assume that there is a $g\left(\neq g_{0}\right) \in G$ such that $(R \mid)_{*}: H_{1}(g) \rightarrow H_{1}\left(g_{0}\right)$ is not an isomorphism. Then, since $H_{1}(N)=\mathbb{Z}_{2},(R \mid)_{*}: H_{1}(g) \rightarrow H_{1}\left(g_{0}\right)$ is trivial. Take the covering $q: M^{*} \rightarrow p^{-1}\left(C^{\prime}\right)$ corresponding to $R_{\#}^{-1}(H)$, where $H=\bigcap_{i \in I} H_{i}$ with $I=\left\{i:\left[\pi_{1}(N): H_{i}\right]=2\right\}$. Then we see that for all $g\left(\neq g_{0}\right) \in G, q^{-1}(g)$ has two components which are homeomorphic to $N$ and $q^{-1}\left(g_{0}\right) \sim N_{H}$, where $N_{H}$ is the covering space of $N$ corresponding to $H$.

Since $\pi_{1}\left(g_{0}\right) /(R \mid)_{\#}\left(\pi_{1}(g)\right)$ is cyclic (and so abelian) and $H$ is the commutator subgroup of $\pi_{1}(N),(R \mid)_{\#}\left(\pi_{1}(g)\right)$ contains $H$. So we have $(R \mid)_{\#}\left(\pi_{1}(g)\right)$ $=H$, because $H_{1}(N)=\mathbb{Z}_{2}$ and $(R \mid)_{\#}$ is not an epimorphism. Hence, by the fact that $H=(q \mid)_{\#}\left(q^{-1}\left(g_{0}\right)\right)$, we have the lifting $\overline{R \mid}$ of $R \mid$ so that $(q \mid) \circ \overline{R \mid}=R \mid$. Hence we have an epimorphism $\mathbb{Z}_{2} \cong H_{1}(N)=H_{1}(g) \rightarrow$ $H_{1}\left(q^{-1}\left(g_{0}\right)\right) \cong H_{1}\left(N_{H}\right)$. So $H_{1}\left(N_{H}\right)$ is either trivial or $\mathbb{Z}_{2}$.

CASE 1: $H_{1}\left(N_{H}\right)$ is trivial. From the homology exact sequence

$$
\mathbb{Z} \cong H_{2}\left(M^{*}, M^{*} \backslash q^{-1}\left(g_{0}\right)\right) \rightarrow H_{1}\left(M^{*} \backslash q^{-1}\left(g_{0}\right)\right) \rightarrow H_{1}\left(M^{*}\right)=0,
$$

we see that $H_{1}\left(M^{*} \backslash q^{-1}\left(g_{0}\right)\right)$ is cyclic. On the other hand, since $(p \circ q)$ : $M^{*} \backslash q^{-1}\left(g_{0}\right)$ is an approximate fibration, we see that $H_{1}\left(M^{*} \backslash q^{-1}\left(g_{0}\right)\right) \cong$ $i_{*}\left(H_{1}\left(q^{-1}(g)_{C}\right)\right) \oplus \mathbb{Z}$ for the inclusion $i: q^{-1}(g)_{C} \rightarrow M^{*} \backslash q^{-1}\left(g_{0}\right)$. By $\left[3\right.$, Theorem 2.5], we deduce that $i_{*}$ is a monomorphism so that $H_{1}\left(M^{*} \backslash\right.$ $\left.q^{-1}\left(g_{0}\right)\right) \cong H_{1}\left(q^{-1}(g)_{C}\right) \oplus \mathbb{Z} \cong \mathbb{Z}_{2} \oplus \mathbb{Z}$, which is not cyclic.

CASE 2: $H_{1}\left(N_{H}\right)=\mathbb{Z}_{2}$. Let $K$ be the commutator subgroup of $\pi_{1}\left(N_{H}\right)=H$. Then $K$ is a normal subgroup of $\pi_{1}(N)$ with index 4. Since $\pi_{1}(N) / K$ is abelian, $K$ contains the commutator subgroup $\pi_{1}\left(N_{H}\right)$ of $\pi_{1}(N)$, which is a contradiction.

Therefore, $p$ is an approximate fibration over int $B$.

Claim (iii). The boundary of $B$ is empty. 
Suppose not. As in the proof of [16, Theorem 3.3], we then could have a map $N \rightarrow N_{H}$ with degree one. But since $H_{1}(N)=\mathbb{Z}_{2}$, we know that $H_{1}\left(N_{H}\right)$ is either trivial or $\mathbb{Z}_{2}$. As in the proof of [3, Lemma 6.7], we see that the case of $H_{1}\left(N_{H}\right)=0$ cannot happen. Also, as before, we can show that the case of $H_{1}\left(N_{H}\right)=\mathbb{Z}_{2}$ cannot happen.

COROLlary 3.3. Let $N^{n}$ be a closed orientable $n$-manifold with hopfian $\pi_{1}(N)$ and $\chi(N) \neq 0$. Then $N$ is a codimension-2 fibrator if $N$ is aspherical or $n=4$.

\section{References}

[1] G. Baumslag and D. Solitar, Some two-generator and one relator non-hopfian groups, Bull. Amer. Math. Soc. 68 (1962), 199-201.

[2] N. Chinen, Manifolds with nonzero Euler characteristic and codimension-2 fibrators, Topology Appl. 86 (1998), 151-167.

[3] - Finite groups and approximate fibrations, ibid., to appear.

[4] D. S. Coram and P. F. Duvall, Approximate fibrations, Rocky Mountain J. Math. 7 (1977), 275-288.

[5] - - - Approximate fibrations and a movability condition for maps, Pacific J. Math. 72 (1977), 41-56.

[6] - - - Mappings from $S^{3}$ to $S^{2}$ whose point inverses have the shape of a circle, Gen. Topology Appl. 10 (1979), 239-246.

[7] R. J. Daverman, Submanifold decompositions that induce approximate fibrations, Topology Appl. 33 (1989), 173-184.

[8] -, Hyperhopfian groups and approximate fibrations, Compositio Math. 86 (1993), $159-176$.

[9] -, Codimension-2 fibrators with finite fundamental groups, Proc. Amer. Math. Soc., to appear.

[10] -, 3-manifolds with geometric structure and approximate fibrations, Indiana Univ. Math. J. 40 (1991), 1451-1469.

[11] J. C. Hausmann, Geometric Hopfian and non-Hopfian situations, in: Lecture Notes in Pure and Appl. Math. 105, Marcel Dekker, New York, 1987, 157-166.

[12] - Fundamental group problems related to Poincaré duality, in: CMS Conf. Proc. 2, Amer. Math. Soc., Providence, R.I., 1982, 327-336.

[13] J. Hempel, 3-manifolds, Ann. of Math. Stud. 86, Princeton Univ. Press, Princeton, N.J., 1976.

[14] R. Hirshon, Some results on direct sum of hopfian groups, Ph.D. Dissertation, Adelphi Univ., 1967.

[15] Y. H. Im, Products of surfaces that induce approximate fibrations, Houston J. Math. 21 (1995), 339-348.

[16] Y. Kim, Strongly hopfian manifolds as codimension-2 fibrators, Topology Appl., to appear.

[17] -, Manifolds with hyperhopfian fundamental group as codimension-2 fibrators, ibid., to appear.

[18] A. N. Parshin and I. R. Shafarevich, Algebra VII, Encyclopaedia Math. Sci. 58, Springer, 1993. 
[19] J. Roitberg, Residually finite, hopfian and co-hopfian spaces, in: Contemp. Math. 37, Amer. Math. Soc., 1985, 131-144.

Department of Mathematics

Pusan National University

Pusan, 609-735, South Korea

E-mail: yhim@hyowon.pusan.ac.kr
Department of Mathematics The University of Tennessee at Knoxville

Knoxville, Tennessee 37996-1300 U.S.A.

E-mail: ykim@math.utk.edu

Received 26 March 1998;

in revised form 26 August 1998 